\title{
Design Optimisation of Air-fed Full Pressurised Suits
}

\author{
P. Edwards, b, (Contact), K. Tesch ${ }^{a, c}$, T.G. Karayiannis ${ }^{a, d}$, M.W. Collins ${ }^{a, d}$, M.A. \\ Atherton $^{\mathrm{a}, \mathrm{d}}$, P. Young ${ }^{\mathrm{a}, \mathrm{e}}$, M. Large $\mathrm{L}^{\mathrm{a}, \mathrm{f}}$ and R. Brade ${ }^{\mathrm{a}, \mathrm{b}}$ \\ a JET-EFDA, Culham Science Centre, OX14 3DB, Abingdon, UK \\ ${ }^{b}$ UKAEA Fusion Association, Culham Science Centre, Abingdon, OX14 3DB, UK \\ c Fluid Mechanics Department, Gdansk University of Technology, Poland \\ ${ }^{d}$ School of Engineering and Design, Brunel University, UK \\ e Defence Science and Technology Laboratories, Porton Down, UK \\ ${ }^{f}$ BNS Nuclear Services, Leicester, UK \\ Contact author - JET-EFDA, Culham Science Centre, OX14 3DB, Abingdon, UK. \\ Tel.: +44 1235 465190; fax: +44 12355315.
}

\begin{abstract}
The JET machine and associated facilities require significant maintenance and enhancement installation activities in support of the experimental exploitation programme. A proportion of these activities are within radiological and respiratory hazardous environments. As such, breathing air-fed one-piece pressurised suits provide workers with protection from the inhalation of both airborne tritium and beryllium dust. The design of these suits has essentially developed empirically. There is a practical necessity to improve the design to optimise worker performance, protection and thermal comfort. This paper details the complexity of modeling the three-dimensional thermofluid domain between the inner surface of the suit and under garments that includes mass as well as heat transfer, suiting geometry, human metabolism and respiration and effects of limb movements. The methods used include computational fluid dynamics (CFD), theoretical adaptations of mixed-phase turbulent flow, profile scanning of a suit and actuating life size mannequin and data processing of the images and experimental validation trials. The achievements of the current programme and collaborations are presented in the paper and future endeavors are discussed.
\end{abstract}

KEYWORDS: Pressurised-suit; Scanning; Respirator protection; CFD

\section{I NTRODUCTI ON}

During engineering interventions of the JET machine significant work is carried out by operators wearing full pressurised suits (Figure 4) in a number of different facilities. In the most recent years the 2004 (EP1 part1) and 2007 (EP1 part2) shutdowns resulted in 3100 and 850 man-hours of work in radiological and hazardous environments. 
The suit is constructed from impermeable polyurethane (PU), which is known for its high evaporative resistance. This quality resists the sweating mechanism so preventing sufficient heat being lost to the environment and only allowing sweat from the body to pass into the micro-climate. As a consequence any excess heat produced due to work and/or the surrounding climate cannot be transferred from the body. This can result in heat stress and in extreme circumstances heat exhaustion. $100 \%$ dry, chilled breathing air reaches the suit through an umbilical attached by a self-sealing coupling in the vicinity of the operators' lumbar area at $390 \mathrm{l} / \mathrm{min}$. An internal suit bulk-head channels this air through tubing to the wrists, feet and head. The suit has two pairs of filtered outlets positioned at the back of the head and buttocks area. Other features include foot protection worn over the suit, rubber gloves attached hermetically to the cuffs and a sacrificial over-suit covering the trunk, arms and legs to avoid contaminating the main suit fabric.

The three-dimensional thermofluids problem includes mass as well as heat transfer. The fluid field is complex bounded on one side by a human body with multi-layer porous cotton under garments and the impermeable polyurethane suit material. Accurate threedimensional geometry scans of a mannequin and full pressurised suit provide the fluid boundary data to the CFD software. The 'Porton Animated Mannequin' is a life size mannequin which is capable of simulating a walking and marching motion enabling a dynamic study of the cooling flow regime to be undertaken. The mannequin consists of a rigid torso attached by the head to the support frame. The mannequin's limbs can only be moved in one plane of motion. With the use of additional supports the arms and legs can be positioned statically in a variety of positions of particular interest (kneeling, squatting, etc.).

\section{DEFI NITION OF THE FLOW DOMAI N}

Photogrammetry [1] is a non-contact surveying technique using photographic images. Multiple photographs of the object are taken from different angles allowing common points to be identified on each image. A line of sight can then be constructed from the camera location to the point on the object. The intersection determines the threedimensional location of the point. To allow the software to identify specific points on the object, small round metallic dots known as targets are attached to the object.

In addition to attaching targets to an object, arrays of virtual targets can be projected onto the object through the use of Geodetic Systems' Pro-Spot [2]. As well as targets, codes are also attached to the object. Codes are a specific arrangement of eight dots that provide the software with greater positional information. The high level of 
automation within the software enabled many different pose models to be produced in a short time.

In order to measure the volume and displacement between the mannequin and the pressurised suit in any given pose within the support frame the following objectives were undertaken.

- Measure and create CAD model of head and torso as fixed within frame.

- Measure and create CAD model of upper arms, forearms, thighs and lower legs.

- Create and qualify accuracy of animation of fixed torso and moving limbs by real time measurement of single coded targets attached to each index finger and big toe of the four limb extremes.

- Measure pressurised suit fitted over mannequin and derive limb positions within suit from the four exposed coded targets fitted to the limb extremes (Figure 1).

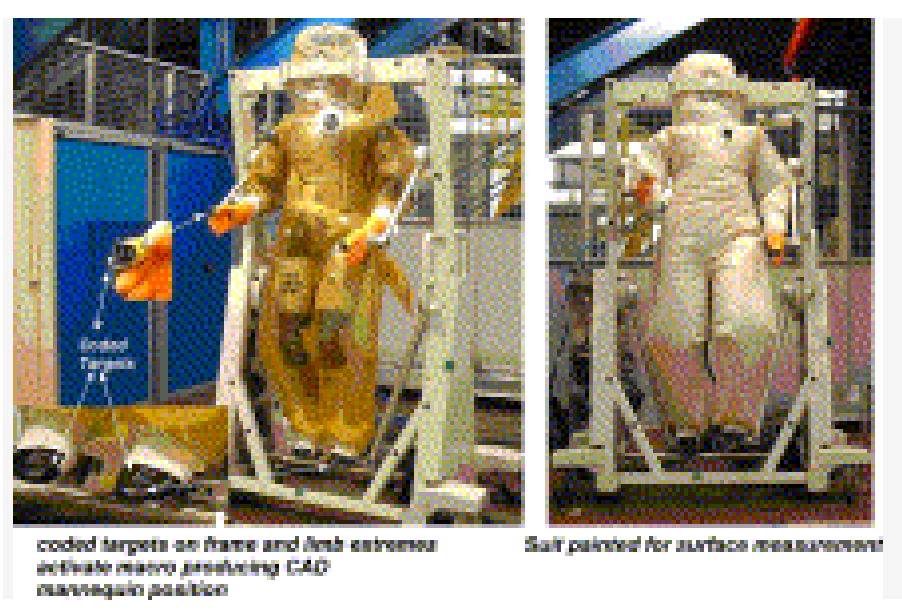

Figure 1: Left: Coded targets on frame and limb extremes active macro producing CAD mannequin position. Right: Suit painted for surface measurement.

- Supply CAD models of body and suit within a simultaneous single pose to enable volumetric measurement of clearance (Figure 2).

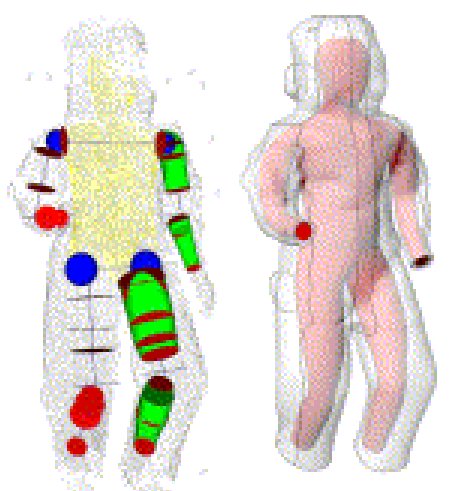


Figure 2: Modeling the flow domain

Measurements of the head and torso proved the frame was adequately rigid to better than $1 \mathrm{~mm}$ throughout all conditions of measurement. Photogrammetry target measurements were recorded at approximately $50 \mathrm{~mm}$ horizontal cross-sections of head and torso. The finished CAD model was then associated with coded targets permanently attached to the frame so that whenever the coded frame targets were measured within V-Stars [3] processing software then the torso model appeared within its correct 3D position. The pivot points at the shoulders and hips were also defined within this fixed model.

Eight individual limb template models were created using the same techniques as previously detailed. The CAD models of each limb were subsequently associated with coded targets attached at the limb extremities.

Measurement of the frame codes and the four exposed limb codes enabled a macro in the V-Stars software to position the CAD models of head and torso, forearms and lower leg calves in true position in real time. This was possible by use of two cameras measuring simultaneously at intervals of two seconds on a constantly moving mannequin. The derived animated models were checked in certain static poses against the actual targets that created the virtual animation models and these were concurrent to an accuracy within $2 \mathrm{~mm}$.

Because the fixed pivot points of hips and shoulders were known and the limb extremes (forearms and lower leg calves) measured the software was able to automatically transform the CAD models of the thighs and upper arms into their correct position via common point alignment thereby completing the full animation. The virtual animation models of these parts were concurrent to an accuracy within $3 \mathrm{~mm}$.

The measurement of the suit (and torso) was carried out using ProSpot. To visualise the virtual targets the clear suit had to be made opaque by the application of a suitable coating. This opaque surface also made it impossible to see any targets on the mannequin and is why the aforementioned limb extreme coded target macros were devised. Initially it was assumed that the inflated suit would be unstable and need real time measurements caught with projected targets and multiple cameras operating simultaneously. Because of a lack of cameras and projectors this would have limited measurement to a front or back but no complete coverage. However tests showed that 
any pose held statically resulted in virtually instant stabilisation of the pressure suit enabling the projector and camera to rotate around the suit giving complete coverage.

\section{THERMOFLUI DS MODEL}

The flow regime is turbulent and consists of two components due to the presence of water vapour through respiration and moisture produced by the human body. The unknowns of the model are the three velocity vectors $U, V, W$, pressure $p$, temperature $T$ and the effective viscosity $\mu_{\mathrm{e}}$. These are solved using ANSYS-CFX a commercial CFD package (in excess of 270,000 nodes) (Figure 3) by defining a set of boundary conditions and coefficients that are applied to the conservation of mass, momentum, energy and turbulence models in the form of the non-linear partial differential equations detailed below [4], [5] and [6]

(1) $\nabla \cdot \vec{U}=0 \quad$ Conservation of Mass

(2)

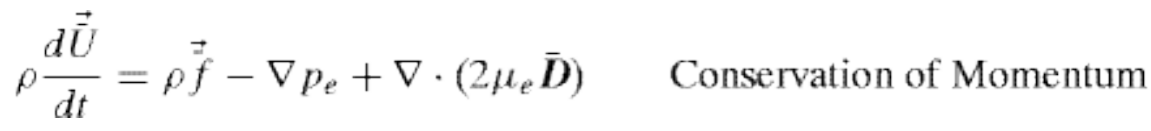

$$
c_{v} \rho \frac{d \bar{T}}{d t}=2 \mu \bar{D}^{2}+\nabla \cdot\left(\lambda_{e} \nabla \bar{T}+\rho \bar{T} \sum_{i=1}^{2} c_{v i} D^{i j} \nabla \tilde{g}^{i}\right)+\rho \varepsilon
$$

(3) Conservation of Energy

$$
\rho \frac{d k}{d t}=2 \mu_{t} \overline{\boldsymbol{D}}^{2}+\nabla \cdot\left(\left(\frac{\mu_{t}}{\sigma_{k}}+\mu\right) \nabla k\right)-\rho \varepsilon
$$

(4) $k$ kinetic energy turbulence model

$$
\rho \frac{d \varepsilon}{d t}=C_{\varepsilon 1} \frac{\varepsilon}{k} 2 \mu_{t} \overline{\boldsymbol{D}}^{2}+\nabla \cdot\left(\left(\frac{\mu_{t}}{\sigma_{\varepsilon}}+\mu\right) \nabla \varepsilon\right)-C_{\varepsilon 2} \rho \frac{\varepsilon^{2}}{k}
$$

(5) $\varepsilon$ dissipation turbulence model

$$
\rho \frac{d \bar{g}^{1}}{d t}=\rho \nabla \cdot\left(D_{e} \nabla \cdot \bar{g}^{1}\right) \quad \text { Moisture transport }
$$


(7) $\bar{g}^{2}=1-\bar{g}^{1} \quad$ Sum of components equals unity
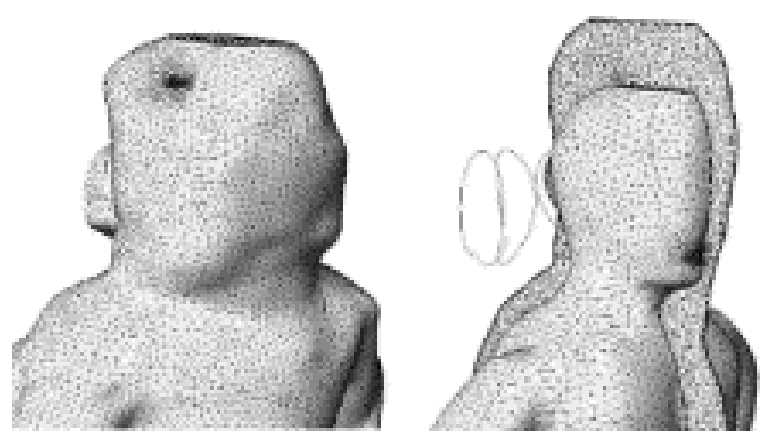

Figure 3: Magnification of the mesh domain

\section{EXPERI MENTAL VALI DATION}

A series of validation trials were instigated (Figure 4) to compare experimental results with the CFD predictions. The breathing air, torso, head, limb, outlet and ambient temperatures and humidity measurements were taken for two configurations. An empty suit enabled the average heat transfer coefficient from the ambient suit side to be determined. A series of experiments at differing ambient temperatures and human metabolic rates (related to the type of work being undertaken) were implemented to enable best-fit values of the average heat transfer coefficient on the body side (Figure $5)$.
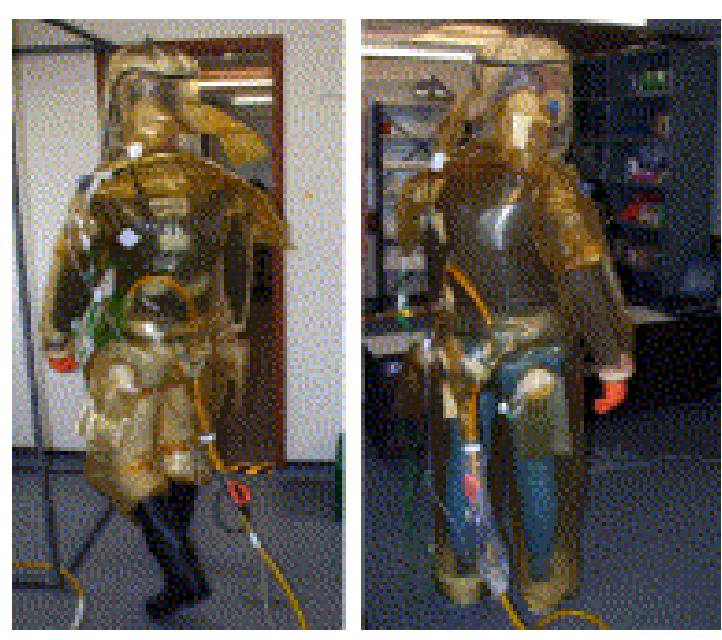

Figure 4: Validation trials, note high length boots 

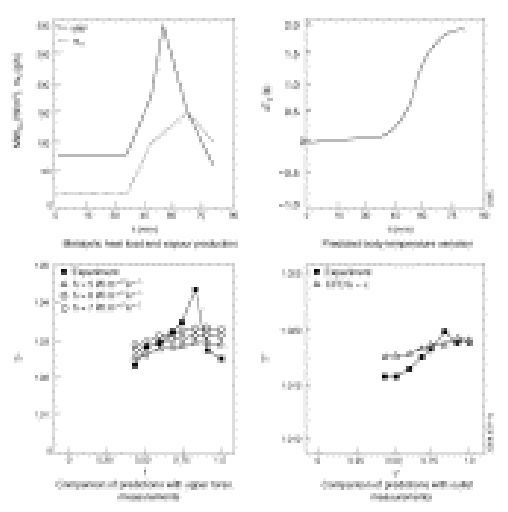

Figure 5: Predictions and measurement comparisons

\section{RESULTS}

Sensitivity analysis identified that the most influential variable is the inlet temperature in terms of the resulting outlet temperature. Breathing air is supplied to the suit through an umbilical which can be from 25 to $50 \mathrm{~m}$ in length. Although chilled at source, the ambient temperature has a large influence on the suit inlet air temperature. Surprisingly the mass flow rate had an insignificant effect on the outlet temperature over the range of $370-410 \mathrm{l} / \mathrm{min}$. In addition changes in the ambient temperature coupled with the low heat transfer coefficient of the suit had little influence (Figure 6).

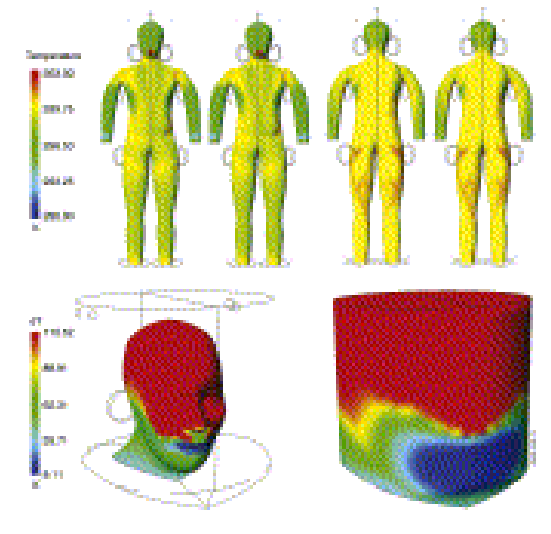

Figure 6: Body temperature profiles, surface and dew temperature differentials

Design parameter checks associated with a number of variations to the standard suit configuration have been analysed. Specifically the operational effects of leg, arm or head inlets being blocked or restricted (the later being due to excessive, tight taping associated with the fitting of additional gloves and the affects of Wellington style boots around the calf area) and also head and buttock outlets blocked due to kneeling, squatting, over-suit flow restriction effects, suit fabric damage, etc., resulting in the redistribution of the remaining mass flow rate. This has identified a potential future suit enhancement of removing the head outlets and repositioning the head inlets towards the back of the helmet resulting in $0.5^{\circ}$ reduction in temperature. However conversely 
blocking or restricting the lower outlets which can occur as a result of improper fitting of the sacrificial over suit or when sitting down leads to an temperature increase compared to the standard configuration.

\section{FUTURE WORK}

Improvement of the CFD predictions is planned with the introduction of variable heat transfer coefficients based on the position/skin/under garment interface, moisture production rates and improved core temperature data. The use of medical codes and facilities including the use of a sweating instrumented mannequin are being investigated.

A CFD dynamic analysis will be carried out with an animated flow domain replicating walking, kneeling and crawling movements. Studies to investigate pressure surges caused by kneeling and the potential for reverse flow from the ambient environment through the outlets or at breaches of the suit fabric and subsequent transport and trajectories of species (water vapour, tritium, and beryllium) within the flow domain will also be undertaken.

Evolutionary optimization (EO) and robust engineering design (RED) principles will be applied to improve the system performance. These techniques involve the simultaneous minimisation and maximisation of functions through the use of mathematical algorithms to arrive at a range of optimum configurations [7] and [8]. Specifically multivariate modeling using the body/suit air gap and other significant parameters as variables. Using this data will enable the identification and implementation of suit enhancements in conjunction with suit manufacturers specifically for the one-piece full pressurised suit used at the Culham Science Centre. Improved links with the respiratory industry may lead to the wider exploitation of the generic codes and techniques established during this project.

\section{ACKNOWLEDGEMENT}

The author gratefully acknowledges the loan of the articulated mannequin from the Defence Science and Technology Laboratories. This work was funded jointly by EPSRC and by the European Communities under the contract of Association between EURATOM and UKAEA. The views and opinions expressed herein do not necessarily reflect those of the European Commission. This work was carried out within the framework of EFDA. 


\section{REFERENCES}

[1]. J. Tait et al, Further developments at JET of remote digital photogrammetry techniques and remote welding under conditions of restricted access, Symposium of Fusion Nuclear Technology, Rome Sept 1999.

[2]. Kubik. D, Greenwood. J., (2003) Development of Photogrammetric Methods of Stress Analysis and Quality Control, Technical Sciences / University of Warmia and Mazury in Olsztyn, Poland Available from:

http://arxiv.org/ftp/physics/papers/0311/0311008.pdf

[3]. B. Macklin et al, The remote photogrammetry survey and engineering analysis of the divertor structure during JET's RTE, B Macklin et al, SOFT 1998, Marseille.

[4]. K.Tesch et al, Thermo-fluid transport Phenomena in air-fed pressurised protective clothing, HEAT 2008.

[5]. K.Tesch et al, Heat and mass transfer in air fed pressurised suits, UK Heat Transfer Conference, Edinburgh, 2007.

[6]. K. Tesch et al, A thermo-fluid model for protective suiting used in fusion reactor shutdown operations, UIT 2008, Palermo.

[7]. K. Tesch et al, Heat transfer coefficient calibrations by means of evolutionary algorithms, ACDM 2008, Bristol.

[8]. M. Atherton et al, Searching for improvement -Information Transfer in Biological Systems, Design in Nature Series Vol. 2. 